\title{
PASADO CERCANO Y FUTURO INMEDIATO DE LA FID/CLA*. UNA VISION A MITAD DEL CAMINO
}

\author{
Humberto Arango \\ Presidente de FID/CLA \\ Director General del Instituto de Documentación e Información Científica y Técnica. \\ La Habana, Cuba
}

\section{En el pasado cercano, el escenario regional}

Recibimos de nuestro estimado colega de la Argentina, Ricardo A. Gietz, en 1988, la Presidencia de FID/CLA. Fue resultado de la voluntad expresada por los miembros nacionales de la organización, pertenecientes a la región, en la Asamblea General celebrada en La Habana durante el Congreso Internacional de Información INFO'88.

Eran tiempos en que la Federación, una vez más, se sostenía básicamente sobre la vocación profesional de sus integrantes, quienes acudian ya fuera de manera institucional o personal en apoyo de sus escasas finanzas.

El drama económico y financiero de la denominada década perdida latinoamericana tuvo sus efectos en la organización argentina que servía de soporte a la Secretaría Permanente de la Comisión y, en general, a la capacidad de las instituciones y personas en los demás países para respaldar por sí mismos acciones bajo el paraguas de FID.

El liderazgo y visibilidad regional que habia tenido FID/CLA en los sesenta y los setenta se había visto disminuido progresivamente y nuevas alternativas, nuevos espacios, emergían para servir de escenario a los esfuerzos sostenidos que llevaba a cabo la vocación de colaboración, cooperación e integración entre los países y subregiones latinoamericanos en el sector informativo y documental.

En los ochenta, predominantemente en su primera mitad, diferentes agencias (UNESCO, FAO, OMS/OPS, ONUDI, OEA, BID, IDRC, CEPAL/CLADES, entre otras) cubrian, con sus programas y apoyo financiero, un espacio cada vez más amplio y diversificado de acciones, las cuales, según las misiones, objetivos, políticas y estrategias de cada agencia influían a nivel de nación, sector, subregión y región en materia de información y documentación.

A mitad de la pasada década la iniciativa INFOLAC constituía, en el plano regional, una alternativa promisoria para facilitar la cooperación y lograr mayor beneficio regional en el empleo efectivo y eficiente de los recursos y capacidades que se estaban creando en diversas redes y sistemas de información. INFOLAC era la identificación del Programa Regional para el Fortalecimiento de la Cooperación entre Redes y Sistemas Nacionales de Información para el Desarrollo en América Latina y el Caribe cuyo objetivo general consistia en:

* Comisión para América Latina de la Federación Internacional de Información y Documentación. 
fortalecer la autonomía y capacidad individual y conjunta de las instituciones nacionales de América Latina y el Caribe para crear y operar mecanismos de organización y acceso al recurso información e intensificar su utilización en la formulación, ejecución, evaluación y administración de sus respectivos planes, programas, políticas y acciones del desarrollo económico, social, científico, tecnológico y cultural. tegias:

INFOLAC se diseñó a partir de los siguientes principios constitutivos y estra-

1. INFOLAC se concibe como un mecanismo de coordinación, cooperación e integración de las actividades de información que realizan las instituciones públicas y privadas, y los organismos regionales e internacionales en la Región Latinoamericana y del Caribe.

2. INFOLAC se plantea, además, como una instancia que estimula el trabajo voluntario «inter pares», y la cooperación horizontal a través del consenso y responsabilidad compartida.

3. Los mecanismos de gestión, coordinación y operación requeridos por tal concepción implican una máxima participación y flexibilidad, con un mínimo de carga administrativa y formalismo. Por otra parte, y vistas las condiciones financieras actuales, 'resulta imperativo que su desarrollo no conlleve la creación de nuevos organismos, siendo más conveniente el fortalecimiento de instituciones, recursos y mecanismos ya existentes.

Entre el 20-22 de octubre de 1988, en La Habana, Cuba, se celebró bajo la convocatoria y auspicio del Centro Internacional de Investigaciones para el Desarrollo de Canadá el Seminario-Taller sobre Experiencias de las Redes Regionales de información en América Latina con el objetivo de reunir juicios evaluativos sobre los resultados alcanzados por estas redes y considerar alternativas sobre acciones posibles a ser desarrolladas con tales actores del sector informativo en la región.

Por esos días del 88, en el mismo Palacio de los Congresos se celebraba la ya mencionada Asamblea General de FID/CLA bajo la presidencia del miembro nacional argentino asumiéndose como estrategia básica para el período 1988-90 lo siguiente:

- las tareas y objetivos de la Comisión de la FID para los países de América Latina se establecen por el reglamento y la disposición para las comisiones regionales de la FID, que son:

- divulgar el programa y la actividad de la FID en la región,

- iniciar, divulgar y mantener el desarrollo de los servicios nacionales de información y documentación en la región,

- estimular y facilitar la colaboración en la esfera de la información y la documentación entre los países de la región y demás países, sobre todo aquellos que gracias a una serie de factores lingüísticos y culturales están más estrechamente relacionados con la región, por ejemplo, España y Portugal, 
- reactivar y fortalecer el funcionamiento de los Comités y Grupos de Trabajo,

- organizar el Congreso regional,

- impulsar la creación de Comités Nacionales de la FID en los países, liderados por el miembro nacional.

Esta estrategia descansaba en decisiones previas adoptadas entre 1984-86 por el Consejo de la FID el cual ya había acordado promover la descentralización en la adopción de decisiones, la planificación y la ejecución de los programas, elevando el papel y la responsabilidad de las Comisiones Regionales.

Como directiva general del Consejo de la FID, la aplicación de los programas se debía descentralizar, en la medida de lo posible, en las Comisiones Regionales. Ello significaba que, progresivamente, se debían ir creando comités y grupos de trabajo en dichas comisiones estableciendo al mismo tiempo relaciones directivas y de coordinación entre los Comités de la FID y los de las regiones. El mayor impedimento para llevar adelante esta estrategia fue, durante toda la década pasada, la carencia de fondos financieros para respaldar este proceso de descentralización.

\section{La FID de los ochenta}

Stella Keenan, Secretaria General de la FID hasta casi finales de la década, expresaba:

La FID reconoce que con los limitados recursos de que dispone no puede atender toda la diversidad de problemas de documentación e información que requieren estudio y adopción de medidas en el marco de sus amplios objetivos. Es necesario establecer una estructura de manera que puedan definirse las esferas $y$ las actividades prioritarias.

En 1978, en su reunión celebrada en Edimburgo, la Asamblea General de la FID aprobó una estructura de programa para la Federación que conformó las bases de sus actividades durante los últimos ocho años. En virtud de esta estructura se elaboró el primer Programa a Plazo Medio para 1981-84. Este programa se basa en cinco prioridades para la acción, que son las siguientes:

- Bases teórico-lingüisticas de la Ciencia de la Información (incluida la terminologia).

- Tratamiento y Tecnología de la Información.

- Educación y Formación de especialistas y usuarios de la información.

- Diseño y Gestión de Sistemas y Redes de Información.

- Necesidades de información y hábitos de los usuarios.

En 1982 se aprobó el segundo Programa a Plazo Medio para 1983-86. En 1984 el Consejo de la FID designó un grupo de programación que lo asesora con respecto a las prioridades de los programas. Al celebrar su nonagésimo aniversario, la FID examina su siguiente programa a plazo medio. Sin emhargo, el Consejo de la FID ha reconocido la necesidad de volver a examinar la 
estructura del programa aprobada en 1978, lo que hará la Asamblea General cuando se reúna durante la $43^{a}$ Conferencia y Congreso en Montreal, en septiembre de 1986.

La ejecución del Programa de la FID descansaba en tres entidades: la Secretaría General, las Comisiones Regionales (América Latina y Asia-Oceanía) y los Comités Técnicos.

A principios de 1986 la FID tenía los Comités Técnicos siguientes:

- Central de Clasificación para la CDU.

- Investigación en la esfera de la Clasificación.

- Terminología de la Información y la Documentación.

- Educación y Formación.

- Informetría.

- Lingüística documentaria.

- Información y Documentación de Patentes.

- Investigación de los Principios Teóricos de la Información Científica.

- Información y Documentación en la Esfera de las Ciencias Sociales.

Entre 1986-90 se inició un proceso de cambios de particular importancia para el futuro de la FID. El Consejo de la FID y las Asambleas Generales de 1988 en Helsinki y de 1990 en la Habana aprobaron sendos informes financieros cada vez más favorables; un necesario enfoque más moderno y dinámico se empezaba a vislumbrar.

El Consejo de la FID tomó la importante decisión de constituir en sociedad civil la infraestructura de FID relacionada con la Clasificación Decimal Universal y ejecutar un plan de acción que guiara el tránsito hacia tal objetivo; todo ello fue posteriormente ratificado por la Asamblea General de la FID.

Los Congresos 44 y 45 de la FID permitieron el encuentro de cerca de 1.400 profesionales de la información y la documentación, así como un mayor impulso a las relaciones y visibilidad de la FID ante importantes organizaciones y personalidades vinculadas al sector gubernamental, intergubernamental y no gubernamental.

Ritva Launo, Presidenta, y Ben G. Goedegebuure, Director Ejecutivo de la FID, califican en el Informe Anual de 1990 este año de importante para el desarrollo de la Federación, durante el cual se ha apreciado un número de cambios relacionados con la administración y dirección de la misma.

Nuevas direcciones han sido iniciadas y nuevas áreas han sido exploradas para desarrollar la organización en los noventa. La FID está ahora en situación saludable, plena del dinamismo requerido para avanzar con confianza hacia su centenario en 1995. La necesaria transformación, para seguir siendo lider en una sociedad de información rápidamente cambiante, ha sido virtualmente completada. Las severas dificultades financieras de los ochenta han sido superadas por la introducción de un estricto control presupuestario y una dirección moderna. El espiritu iniciador y emprendedor, que es esencial en una organización consagrada a la promoción de la Investigación y el Desarrollo, está siendo fortalecido nuevamente. 
A mediados de los ochenta un Plan Estratégico fue presentado y aprohado. Este plan fue básicamente considerado como un catalizador para cambiar $e$ iniciar un proceso de revisión constante de nuestras necesidades, objetivos $y$ prácticas. En 1991 el programa profesional será nuevamente adaptado a los cambios que se hagan necesarios por los desarrollos en la profesión y los requerimientos de los miembros. A la Federación, la base de una sólida membresía y sanas prácticas financieras y administrativas le permitirán adaptabilidad, una dirección flexible $y$ un enfoque empresarial, lo cual es tan esencial en una organizaciión profesional moderna como en una organización comercial.

\section{El miembro nacional de Cuba en los ochenta}

El Instituto de Documentacioin e Información Científica y Técnica (IDICT) ingresa en 1964 como miembro nacional de Cuba en la FID. Este Instituto desde su creación ha ocupado una sostenida posición de liderazgo nacional en el campo de la documentación y la información, teniendo una participación protagónica en el diseño y ejecución de políticas nacionales de información, en la gestación, organización y funcionamiento de la infraestructura de los servicios de información especializada; asi como en el desarrollo de la profesión, los profesionales y los usuarios de la información y la documentación en Cuba.

En 1988, el IDICT conmemoraba su 25 Aniversario y servia la fecha para hacer un recuento del camino seguido en la creación, consolidación y desarrollo de la actividad de documentación e información en el plano nacional.

El nuevo Programa Educacional Cubano había eliminado un millón de analfabetos absolutos, una cifra superior de semianalfabetos; incorporó a la escuela seiscientos mil niños que carecían de ella; llevó a nuestra población, que al triunfo de la Revolución apenas rebasaba los seis millones de habitantes y cuya escolaridad promedio era de tercer grado, a un sistema educacional cuya enseñanza es obligatoria hasta el noveno grado para una población predominantemente joven, que rebasa los diez millones de habitantes.

La economia cubana, estructuralmente deformada, atrasada y dependiente, se transformó con el desarrollo predominante de sus sectores primarios y secundarios. Surgian y se desarrollaban los usuarios institucionales e individuales de la información y la documentación.

Junto a las urgentes tareas de erradicar el analfabetismo, desarrollar la educación, la salud y la industrialización; elevar aceleradamente el desarrollo socioeconómico, cultural y científico-técnico, asi como asegurar la defensa del país, el Gobierno Revolucionario prestó atención, desde los primeros años, a la creación y desarrollo de los servicios de documentación e información científica y técnica y expresó claramente la voluntad politica de establecer las bases del Sistema Nacional de Información, así señalado en el texto de la Ley 1107 del 19 de abril de 1990, que creó el IDICT, adscrito a la entonces Comisión Nacional de la Academia de Ciencias de Cuba.

En 1959, se contaba en Cuba con un exiguo número de bibliotecas especializadas que correspondian fundamentalmente a las universidades existentes, asociaciones profesionales y algunos hospitales y compañias privadas; los recursos hu- 
manos apenas llegaban al centenar de trabajadores; existian muy pocas colecciones básicas, siendo su procesamiento y diseminación prácticamente nulos.

La importancia de la documentación y la información científica y técnica comienza, por tanto, a ser valorada como consecuencia del ímpetu renovador de las radicales transformaciones políticas, económicas y sociales que la revolución imprime a la vida nacional; tal importancia tiene como exponentes principales la creación de centros de información y documentación, desde principios de los sesenta, en los campos de la salud, la agricultura, la industria, la ciencia y la educación incluyendo la creación y desarrollo de redes sectoriales en estos campos.

En 1971, se funda la Licenciatura en Información Cientifico-Técnica y Bibliotecología en la Universidad de la Habana; desde la década del sesenta se dispone de escuelas para la formación de técnicos medios en Bibliotecología.

A finales de los ochenta Cuba estaba dotada de un Sistema Nacional de Información Científica y Técnica que contaba con cerca de 800 unidades de información y más de 7.000 trabajadores, de los cuales alrededor del 45 por 100 eran profesionales universitarios; incluidos los técnicos medios, se alcanza el 70 por 100 del total.

Como parte de la estrategia de desarrollo se encontraba el incremento, ampliación y diversificación del intercambio profesional internacional que habia transcurrido predominantemente con la Unión Soviética y los países de Europa del Este. En 1986, durante el Congreso de la FID en Montreal, Canadá, obtuvo La Habana, por votación y por la diferencia favorable de un voto, la sede del 45 Congreso y Conferencia de la FID.

Como actividad preparatoria y de entrenamiento organizativo se libró la convocatoria al Congreso INFO'88 bajo el lema "Información-Cooperación-Integración» del cual fue escenario el Palacio de Convenciones donde se reunieron más de 650 profesionales, actividad que sirvió de marco a la XXI Asamblea Regional de FID/CLA.

\section{El pasado más cercano, prólogo del futuro inmediato}

La XXII Asamblea General de FID/CLA se celebró en La Habana a finales de 1990 como parte de las actividades realizadas durante el 45 Congreso y Conferencia de la FID; la misma se inscribe en la primera fase del proceso de cambios que, en marcha todavía inicial, ocurría para la Federación y cuyos efectos no se hacian sentir aún en las Comisiones Regionales.

Participaron en la reunión los miembros nacionales de Bolivia, Brasil, Chile, Costa Rica, Cuba, México, Nicaragua y Venezuela; como observadores e invitados, Ecuador, España, CEPAL/CLADES, UNESCO/PGI, INFOLAC, ACURIL y UNESCO/SIIT.

Los debates de esta Asamblea estuvieron centrados en perfilar un nuevo enfoque estratégico de FID/CLA a ejecutar en el corto plazo (1990-92) que partía de la apreciación que se tenía del escenario regional, de la situación de la Federación y de la propia Comisión Regional. En los dos últimos años se habia discutido con el ejecutivo de la FID alternativas para captar financiamiento que habian tenido su aprobación ejecutiva por parte de la Presidencia, el Tesorero y la Secretaría General. 
Las conclusiones principales fueron las siguientes:

- Posicionamiento de FID/CLA como la Comisión Regional para América Latina de la Federación Internacional para la Información y la Documentación, y fortaleciendo su papel como asociación internacional de instituciones y personas que desarrollan, producen, investigan y usan productos informativos, sistemas de información y métodos; así como están involucrados directa o indirectamente en la gestión y dirección de la información.

- Orientación hacia la misión de FID:

Operar y desarrollar de manera continuada, con alcance mundial, programas de información, actividades y redes para servir y apoyar a los modernos profesionales y usuarios de la información.

- Aumentar la visibilidad de FID/CLA en la región; para ello:

- establecer alianzas con programas e instituciones relevantes para la región en materia de información y documentación.

- mantener el boletín "Informaciones FID/CLA».

- mantener la versión española de la Revista de la FID Forum Internacional de Información y Documentación.

- Resaltar el carácter profesional de la FID y, por tanto, de FID/CLA; sus posibilidades como red de expertos que permita promover servicios de consultoría y asistencia técnica para la gestación, desarrollo y evaluación de proyectos.

- Propiciar el intercambio profesional y los contactos institucionales e individuales en la región y aprovechar las oportunidades que brindan eventos tales como: FID’90 (Cuba), ACURIL (1991, Venezuela), FID'92 (España), IFLA (1992, España), INFO’93 (Cuba), IFLA (1994, Cuba).

- Empleo del fax y el correo electrónico para mejorar las comunicaciones entre los miembros de FID/CLA.

- Lograr una nueva imagen de FID/CLA integrada a la imagen corporativa de la FID.

- Elevar el papel del miembro nacional como aglutinador de la comunidad profesional (instituciones, asociaciones y personas) en documentación e información para cada país, constituyendo los resultados en esta dirección un indicador de liderazgo nacional y de papel activo en las tareas de la Federación.

Entre 1990-92 en mayor o menor medida se lograron avances en casi todas las direcciones de FID/CLA. En este tiempo la FID continuó acelerando sus cambios hasta constituirse en conducta permanente un estilo flexible, dinámico y moderno de dirección.

La reunión del Comité Ejecutivo de la FID en abril del 91 en La Haya abría el camino hacia un sugerente ejercicio de planeamiento estratégico en cuyo inicio no se perfilaba aún el papel de las Comisiones Regionales; las reuniones posteriores de Washington y Oslo las identificaron como una de las fortalezas de la Federación y sobre esta premisa será necesario trabajar para el perfeccionamiento de las mismas. 
La alianza FID/CLA-UNESCO-INFOLAC, la cual se consideró prioritaria en la XXII Assamblea General de FID/CLA, se llevó adelante y se refleja en el apartado 5 del artículo "Las políticas de información de la UNESCO y su repercusión en América Latina» que se publica en este mismo número.

En agosto de 1990, la Asociación Latinoamericana de Instituciones Financieras de Desarrollo (ALIDE), el Centro Internacional de Investigaciones para el Desarrollo (CIID, Canadá), el Programa Latinoamericano y del Caribe de Información Comercial y de Apoyo al Comercio Exterior (PLACIEX) y el Centro Panamericano de Ingeniería Sanitaria y Ciencias del Ambiente (CEPIS) organizaron la celebración en Lima, Perú, del Seminario sobre Red de Redes Latinoamericanas de Información.

Tomado de su informe final señalamos Objetivos y Temario:

\section{Objetivos}

En seguimiento a las conclusiones y recomendaciones del Seminario-Taller sobre Experiencias de las Redes Regionales de Información en América Latina realizado del 20 al 22 de octubre de 1988 en La Habana, Cuba, y en respuesta a la dinámica propia del desarrollo de las redes de información, así como a los avances tecnológicos en materia de transmisión de información y comunicaciones, el Seminario tuvo por objetivo general fortalecer las relaciones de cooperación entre las redes de información regionales en sus respectivos campos de actuación, para lo cual se examinaron y debatieron los alcances de una Red de Redes Latinoamericanas de Información, orientada a optimizar la utilización regional de recursos técnicos y tecnológicos de cada red en beneficio del servicio que éstas brindan a sus usuarios.

Más en particular, el Seminario tuvo como propósito analizar soluciones técnicas y otras a requerimientos y problemas de las redes de información, así como los lineamientos de un Proyecto para la conformación de una Red de Redes definiendo sus objetivos, alcances y áreas de actuación metodológica.

- Continuar trabajando en el perfeccionamiento del Boletín de la Comisión, en aras de lograr una publicación cada vez más profesional, desde todos los puntos de vista y que satisfaga cada vez mejor los objetivos para los cuales fue creado.

- Lograr como propósito central de la Comisión en este periodo:

- Llevar a cabo la misión, los objetivos y los programas de la Federación, asumiendo la región como segmentación geográfica del mercado global que abarca la FID.

- A partir de lo anterior preparar ideas, proposiciones y proyectos sobre FID/CLA para su consideración por la Comisión Regional, el Comité Ejecutivo y el Consejo de la FID relacionados con:

* Fortalezas, debilidades, oportunidades y amenanzas.

* Planeamiento estratégico.

* Programa profesional. 
* Membresía.

* Finanzas.

* Diseño organizacional.

* Orientación al mercado.

* Relaciones de colaboración y alianzas.

* Imagen.

* Publicaciones.

* Visibilidad.

\section{Bibliografia}

1. ALIDE. Seminario sobre Red de Redes Latinoamericanas de Información, Lima, Perú, 14-17 de agosto de 1990. Informe final, 1990.

2. ARANGO SALES, H. Servicios de Información especializada para el desarrollo: Cuba. Su experiencia, Ciencias de la Información (La Habana), 23 (1), 10-14 de marzo de 1992.

3. ARANGO SALES, H. Discurso de apertura de la III Jornada Científica del IDICT. Actualidades de la Información Cientifico-Técnica (La Habana), 3 (140), 1988.

4. CRUZ RAMOS, R., y ZETTER-LEAL, J. Las politicas de información de la UNESCO y su repercusión en América Latina: ponencia en el 46 Congreso de la FID, octubre 1992 (publicado en este mismo número).

5. FID. International Federation for Information and Documentation. Annual Report 1990. FID 696, ISSN 0303-4542.

6. FID. Rules of Procedure Regional Commissions. Documento interno.

7. FID/CLA. Informe resumen sobre la actividad de la Comisión Regional en el período 1991-92, octubre 1992.

8. FID/CLA. Actas de la XXII Asamblea General de la Comisión Regional para América Latina de la FID, septiembre 1990.

9. GIETZ, R. A. Comisión de la FID para los paises de América Latina (FID/CLA), Forum Internacional de Información y Documentación, 11 (3), 31-33, julio 1986.

10. GIETZ, R. A. Comisión de la FID para los países de América Latina y su actividad, International Forum of Information and Documentation, 8 (4), 32-34, 1983.

11. GIETZ, R. A. Reflexiones sobre FID/CLA. Informe presentado a la XXII Asamblea General de FID/CLA, septiembre 1990 (no publicado).

12. HILL, M. W. Discurso de Apertura en el XLIV Congreso de la FID, Forum Internacional de Información y Documentación, 14 (1), 33-34, 1989.

13. INFOLAC. Informe final de la reunión sobre "Los lineamientos y puesta en marcha de un programa regional para el fortalecimiento de la cooperación entre redes y sistemas nacionales de información para el desarrollo en América Latina y el Caribe", Santiago de Chile, 3-7 noviembre 1986, CEPAL/CLADES-UNESCO/PGI, 1987.

14. INFOLAC. Lineamientos de un Programa Regional para el fortalecimiento de la cooperación entre redes y sistemas nacionales de información para el desarrollo en América Latina y el Caribe, CEPAL/CLADES-UNESCO/PGI.

15. KEFNAN, S. La FID a los noventa años. Forum Internacional de Información y Documentación, 11 (3), 25-30, julio 1986.

16. KING, A. La gran transición, Forum Internacional de Información y Documentación. $14(2), 3-8,1989$.

17. PRAT Trabal, A. M. Análisis Comparativo de Redes de Información de América Latina y el Caribe. Informe presentado al Seminario-Taller sobre Experiencias de las Redes Regionales de Información en América Latina, La Habana, octubre 1988. 